\title{
Correction \\ Correction: Gill, R.D. Does Geometric Algebra Provide a Loophole to Bell's Theorem? Entropy 2020, 22, 61
}

\author{
Richard David Gill (1)
}

check for updates

Citation: Gill, R.D. Correction: Gill, R.D. Does Geometric Algebra Provide a Loophole to Bell's Theorem?

Entropy 2020, 22, 61. Entropy 2021, 23, 631. https://doi.org/10.3390/ e23050631

Received: 21 April 2021

Accepted: 8 May 2021

Published: 19 May 2021

Publisher's Note: MDPI stays neutral with regard to jurisdictional claims in published maps and institutional affiliations.

Copyright: (C) 2021 by the author. Licensee MDPI, Basel, Switzerland. This article is an open access article distributed under the terms and conditions of the Creative Commons Attribution (CC BY) license (https:/ / creativecommons.org/licenses/by/ $4.0 /)$.
Mathematical Institute, Leiden University, Rapenburg 70, 2311 EZ Leiden, The Netherlands; gill@math.leidenuniv.nl

Corrections are made to my paper "Gill, R.D. Does Geometric Algebra Provide a Loophole to Bell's Theorem? Entropy 2020, 22, 61". Firstly, there was an obvious and easily corrected mathematical error at the end of Section 6 of the paper. In the Clifford algebra under consideration, the basis bivectors $M e_{i}$ do not square to the identity, but to minus the identity. However, the trivector $M$ does square to the identity and hence non-zero divisors of zero, $M-1$ and $M+1$, can be found by the same argument as was given in the paper.

Secondly, in response to a complaint about ad hominem and ad verecundam arguments, a number of scientifically superfluous but insulting sentences have been deleted, and other disrespectful remarks have been rendered neutral by omission of derogatory adjectives. I would like to apologize to Dr. Joy Christian for unwarranted offence.

The end of Section 6 of Gill (2020) [1] discussed the even sub-algebra of $\mathcal{C} \ell_{4,0}$, isomorphic to $\mathcal{C} \ell_{0,3}$ :

One can take as basis for the eight-dimensional real vector space $\mathcal{C} \ell_{0,3}$ the scalar 1 , three anti-commuting vectors $e_{i}$, three bivectors $v_{i}$, and the pseudo-scalar $M=e_{1} e_{2} e_{3}$. The algebra multiplication is associative and unitary (there exists a multiplicative unit, 1). The pseudo-scalar $M$ squares to -1 . Scalar and pseudoscalar commute with everything. The three basis vectors $e_{i}$, by definition, square to -1 . The three basis bivectors $v_{i}=M e_{i}$ square to +1 . Take any unit bivector $v$. It satisfies $v^{2}=1$ hence $v^{2}-1=(v-1)(v+1)=0$. If the space could be given a norm such that the norm of a product is the product of the norms, we would have $\|v-1\| \cdot\|v+1\|=0$ hence either $\|v-1\|=0$ or $\|v+1\|=0$ (or both), implying that either $v-1=0$ or $v+1=0$ (or both), implying that $v=1$ or $v=-1$, neither of which are true.

But the bivectors $v_{i}$ square to -1 and the trivector $M$ squares to +1 . Still, it then follows that $(M+1)(M-1)=0$, and by the argument originally given, it follows that $M=1$ or $M=-1$, a contradiction.

\section{Reference}

1. Gill, R.D. Does Geometric Algebra Provide a Loophole to Bell's Theorem? Entropy 2020, 22, 61. [CrossRef] [PubMed] 